\title{
Two-Dimensional Correlation of Isotropic and Directional Diffusion Using NMR
}

\author{
João P. de Almeida Martins* and Daniel Topgaard \\ Division of Physical Chemistry, Department of Chemistry, Lund University, 22100 Lund, Sweden
}

(Received 23 September 2015; published 23 February 2016)

\begin{abstract}
Diffusion nuclear magnetic resonance (NMR) is a powerful technique for studying porous media, but yields ambiguous results when the sample comprises multiple regions with different pore sizes, shapes, and orientations. Inspired by solid-state NMR techniques for correlating isotropic and anisotropic chemical shifts, we propose a diffusion NMR method to resolve said ambiguity. Numerical data inversion relies on sparse representation of the data in a basis of radial and axial diffusivities. Experiments are performed on a composite sample with a cell suspension and a liquid crystal.
\end{abstract}

DOI: 10.1103/PhysRevLett.116.087601

Many porous materials of biological, geological, and synthetic origin contain water in a range of microscopic environments with different local pore geometries. Information about the structure of the pore space can be inferred from nuclear magnetic resonance (NMR) and magnetic resonance imaging (MRI) measurements of the self-diffusion of the pore water [1,2]. The diffusion MRI approach has been especially powerful for noninvasive studies of the living human brain [3], allowing for quantification of axon diameter [4], mean orientation [5], and orientation distribution [6]. Although useful, classical diffusion MRI protocols relying on the Stejskal-Tanner experiment [7] suffer from the fact that the effects of distributions in pore size, anisotropy, and orientation are intrinsically entangled. A partial solution to this problem is provided by the double diffusion encoding (DDE) family of NMR methods [8], which can give estimates of the pore size and shape even in the presence of orientational disorder [9-23]. Current in vivo versions of DDE permit detection of anisotropy in areas of the human brain that are macroscopically isotropic [24] and the assignment of metabolitespecific compartment shapes in animal models [25]. Despite these impressive feats, DDE yields ambiguous results if the investigated volume element comprises several types of water environments, the presence of which has been inferred by fitting multicomponent biophysical models [26-29] to in vivo data acquired with the StejskalTanner method [30,31]. Selection of a single model from all the ones that are able to reproduce the experimental data remains a challenge [29]. The key to future progress in diffusion NMR and MRI of heterogeneous anisotropic materials lies in designing a method to unambiguously resolve and quantify water compartments with respect to

Published by the American Physical Society under the terms of the Creative Commons Attribution 3.0 License. Further distribution of this work must maintain attribution to the author(s) and the published article's title, journal citation, and DOI. their size and anisotropy, irrespective of the details of their orientations. Once this goal has been achieved, the obtained information could be used as input for existing methods to estimate distributions of axon diameters [4] and orientations [32-34].

In solid-state NMR spectroscopy [35], the eigenvalues and eigenvectors of the chemical shift tensors can be determined through the dependence of the nuclear spin Hamiltonian on the orientation of the tensors with respect to the static magnetic field. We have recently pointed out that the interaction between chemical shift tensors and the magnetic field vector is analogous to the interaction between diffusion tensors and the time integral of the magnetic field gradient vector in diffusion NMR [36]. Assuming that the water molecules in a porous material can be grouped into subensembles, each of which being associated with its own specific microscopic diffusion tensor, then the field of solid-state NMR can serve as a rich source of inspiration when designing new diffusion NMR and MRI methods [36-38]. Even though all the details of restricted diffusion in a porous material cannot be captured within a tensor model, the multitensor approximation is a useful point of departure for the analysis of data from heterogeneous porous materials $[6,13,30]$. In the case of restricted diffusion, the diffusion tensor should be interpreted as an apparent one, being given by the pore size and shape, the local diffusivity within the pore space, as well as the timing parameters of the used pulse sequence [39-44]. A more in-depth analysis of the validity of this approximation is given in the Supplemental Material [45].

Here, we propose a diffusion NMR experiment to resolve distinct water components using inspiration from 2D solid-state NMR techniques correlating isotropic and anisotropic chemical shifts [50-52]. In these techniques, the NMR signal is sampled in a $2 \mathrm{D}$ time-domain space $\left(t_{1}, t_{2}\right)$ where the spins evolve under both isotropic and anisotropic chemical shifts in the first time dimension $t_{1}$, and exclusively under the isotropic chemical shift in the second dimension $t_{2}$. The time-domain signal $S\left(t_{1}, t_{2}\right)$ is 
related to the frequency-domain spectrum $I\left(\omega_{1}, \omega_{2}\right)$ via a 2D Fourier transformation:

$$
S\left(t_{1}, t_{2}\right)=\int_{-\infty}^{\infty} \int_{-\infty}^{\infty} I\left(\omega_{1}, \omega_{2}\right) e^{i \omega_{1} t_{1}} e^{i \omega_{2} t_{2}} d \omega_{1} d \omega_{2} .
$$

When the signal is sampled on a rectangular grid in $\left(t_{1}, t_{2}\right)$ space, then $I\left(\omega_{1}, \omega_{2}\right)$ can be directly estimated with the fast Fourier transformation algorithm. The $\omega_{2}$ dimension of $I\left(\omega_{1}, \omega_{2}\right)$ provides resolution of sites with distinct isotropic chemical shifts, while the $\omega_{1}$ dimension gives information about the chemical shift anisotropy for each of the resolved sites. In our diffusion NMR version, we resolve subensembles of water using their distinct isotropic diffusivities, and, for each of the subensembles, quantify their microscopic diffusion anisotropy.

Consider a material consisting of multiple microscopic domains where water diffusion can be approximated as being Gaussian and described by a microscopic diffusion tensor D. The diffusion NMR signal amplitude is given by $[1,2]$

$$
S(\mathbf{b})=S_{0}\left\langle e^{-\mathbf{b}: \mathbf{D}}\right\rangle,
$$

where $S_{0}$ is the signal obtained when the diffusion-encoding magnetic field gradients have zero amplitude, $\mathbf{b}$ and $\mathbf{D}$ are, respectively, the diffusion-weighting and diffusion tensors, which both are symmetric positive-definite second-order tensors, $\langle\cdot\rangle$ denotes a population-weighted average over the domains, and $\mathbf{b}: \mathbf{D}$ symbolizes a generalized scalar product defined as $\mathbf{b}: \mathbf{D}=\sum_{i} \sum_{j} b_{i j} D_{i j}$. The size, shape, and orientation of the $b$ tensor are under direct experimental control through their dependence on the applied magnetic field gradients $[38,53,54]$. The diagonalized $b$ tensor can be decomposed into its linear $b_{\mathrm{L}}$, planar $b_{\mathrm{P}}$, and spherical $b_{\mathrm{S}}$ components according to

$\mathbf{b}=b_{\mathrm{L}}\left[\begin{array}{lll}0 & 0 & 0 \\ 0 & 0 & 0 \\ 0 & 0 & 1\end{array}\right]+\frac{b_{\mathrm{P}}}{2}\left[\begin{array}{lll}0 & 0 & 0 \\ 0 & 1 & 0 \\ 0 & 0 & 1\end{array}\right]+\frac{b_{\mathrm{S}}}{3}\left[\begin{array}{lll}1 & 0 & 0 \\ 0 & 1 & 0 \\ 0 & 0 & 1\end{array}\right]$.

In order to keep the analogy with Eq. (1), we consider only the case $b_{\mathrm{P}}=0$. With this parametrization of the $b$ tensor, Eq. (2) can be expressed as

$$
\begin{aligned}
& S\left(b_{\mathrm{L}}, b_{\mathrm{S}}\right) \\
& =S_{0} \int_{0}^{\infty} \int_{0}^{\infty} P\left(D_{z z}, D_{\text {iso }}\right) e^{-b_{\mathrm{L}} D_{z z}} e^{-b_{\mathrm{S}} D_{\text {iso }}} d D_{z z} d D_{\text {iso }},
\end{aligned}
$$

where $D_{\text {iso }}=\operatorname{trace}(\mathbf{D}) / 3$ is the isotropic diffusivity and $D_{z z}$ is the $z z$ element of $\mathbf{D}$ in the principal axis system (PAS) of the $b$ tensor. The function $P\left(D_{z z}, D_{\text {iso }}\right)$ denotes the joint probability density of water being in a microscopic environment with the diffusivities $D_{z z}$ and $D_{\text {iso }}$. Equation (4) closely resembles Eq. (1), the main difference being the real rather than imaginary arguments of the exponential functions. In the analogy between Eqs. (1) and (4), $P\left(D_{z z}, D_{\text {iso }}\right)$ assumes the role of $I\left(\omega_{1}, \omega_{2}\right)$. The signal $S\left(b_{\mathrm{L}}, b_{\mathrm{S}}\right)$ is the 2D Laplace transformation of the distribution $P\left(D_{z z}, D_{\text {iso }}\right)$, which in principle can be extracted by applying a 2D inverse Laplace transformation (ILT) [55-57] to data acquired as a function of $b_{\mathrm{L}}$ and $b_{\mathrm{S}}$.

An isotropic orientation distribution of axially symmetric diffusion tensors, with radial and axial diffusivities $D_{\perp}$ and $D_{\|}$, respectively, gives rise to a $2 \mathrm{D}$ line shape

$$
\begin{aligned}
& L\left(D_{z z}, D_{\text {iso }}, D_{\perp}, D_{\|}\right) \\
& =\delta\left[D_{\text {iso }}-\left(2 D_{\|}+D_{\perp}\right) / 3\right] \\
& \quad \times \int_{0}^{\pi / 2} \delta\left[D_{z z}-\left(D_{\|} \cos ^{2} \theta+D_{\perp} \sin ^{2} \theta\right)\right] \sin \theta d \theta,
\end{aligned}
$$

in the distribution $P\left(D_{z z}, D_{\text {iso }}\right)$. In Eq. (5), $\delta[\cdot]$ is the Dirac delta function and $\theta$ is the polar angle of the diffusion tensor $z$ axis in the $b$ tensor PAS. The expression in Eq. (5) corresponds to the $2 \mathrm{D}$ line shape for an axially symmetric chemical shift tensor in the solid-state NMR experiments for correlating isotropic and anisotropic chemical shifts [50-52].

According to Eq. (5), each component with specific values of $D_{\perp}$ and $D_{\|}$gives rise to a ridge in the 2D distribution $P\left(D_{z z}, D_{\text {iso }}\right)$. A collection of such ridges is challenging to reproduce with the standard 2D ILT algorithms due to inversion artifacts $[12,58]$. In order to circumvent this problem, we introduce the distribution $P\left(D_{\perp}, D_{\|}\right)$where each distinct component gives rise to a 2D delta function. The $\left(D_{\perp}, D_{\|}\right)$basis thus offers a sparse representation of the data. The distributions $P\left(D_{z z}, D_{\text {iso }}\right)$ and $P\left(D_{\perp}, D_{\|}\right)$are related via the integral transformation

$$
\begin{aligned}
& P\left(D_{z z}, D_{\text {iso }}\right) \\
& \quad=\int_{0}^{\infty} \int_{0}^{\infty} L\left(D_{z z}, D_{\text {iso }}, D_{\perp}, D_{\|}\right) P\left(D_{\perp}, D_{\|}\right) d D_{\perp} d D_{\|} .
\end{aligned}
$$

Insertion of Eq. (6) into Eq. (4) yields

$$
\begin{aligned}
& S\left(b_{\mathrm{L}}, b_{\mathrm{S}}\right) \\
& =S_{0} \int_{0}^{\infty} \int_{0}^{\infty} K\left(b_{\mathrm{L}}, b_{\mathrm{S}}, D_{\perp}, D_{\|}\right) P\left(D_{\perp}, D_{\|}\right) d D_{\perp} d D_{\|},
\end{aligned}
$$

where 


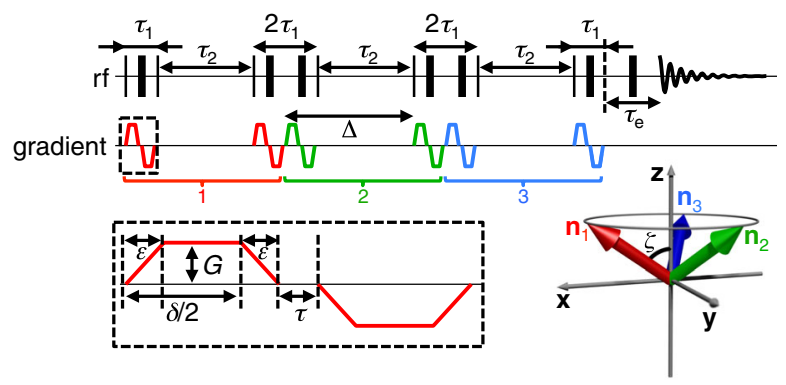

FIG. 1. NMR pulse sequence for correlating isotropic and directional diffusion. The series of $90^{\circ}{ }_{x}$ (thin vertical line) and $180^{\circ}$ (thick vertical lines) radiofrequency (rf) pulses gives rise to a signal (decaying sinusoidal), the amplitude of which is encoded for diffusion using three sets of ramped gradient pulses as indicated with the numbered and colored braces. The dashed box shows a magnification of the first bipolar gradient pulse. The gradient amplitude $G$ and the time intervals $\delta, \Delta, \varepsilon$, and $\tau$ enter the calculation of the trace of the $b$ tensor in Eq. (9). The bottom right panel illustrates the unit vectors $\left(\mathbf{n}_{1}, \mathbf{n}_{2}, \mathbf{n}_{3}\right)$ of the three sets of gradient pulses. The vectors form the angle $\zeta$ with the $z$ axis and are located with threefold symmetry on a right circular cone with apex at the origin. (Adapted with permission from Ref. [59]. Copyright 2015 by Elsevier.)

$$
\begin{aligned}
K\left(b_{\mathrm{L}}, b_{\mathrm{S}}, D_{\perp}, D_{\|}\right)= & \frac{\sqrt{\pi} e^{-b_{\mathrm{L}} D_{\perp}} \operatorname{erf}\left(\sqrt{b_{\mathrm{L}}\left(D_{\|}-D_{\perp}\right)}\right)}{2 \sqrt{b_{\mathrm{L}}\left(D_{\|}-D_{\perp}\right)}} \\
& \times e^{-b_{\mathrm{S}} \frac{D_{\|}+2 D_{\perp}}{3}} .
\end{aligned}
$$

The distribution $P\left(D_{\perp}, D_{\|}\right)$can be estimated by inversion of Eq. (7) using numerical approaches similar to the ones used for the 2D ILT [55].

We have recently introduced the pulse sequence in Fig. 1, which has the capability to sample a range of shapes of the $b$ tensor [59]. The signal is encoded for diffusion in a sequence of three directions $\mathbf{n}_{1}, \mathbf{n}_{2}$, and $\mathbf{n}_{3}$, defined by three azimuthal angles consecutively rotated by $2 \pi / 3$ radians, and a constant polar angle $\zeta$. The trace of the $b$ tensor, corresponding to the $b$ value in conventional diffusion NMR, is given by [59]

$b=3(\gamma G \delta)^{2}\left(\Delta-\delta / 3-\tau / 2-\varepsilon / 2-\varepsilon^{2} / 6 \delta+\varepsilon^{3} / 15 \delta^{2}\right)$,

where $\gamma$ is the gyromagnetic ratio, $G$ is the gradient amplitude, and the timing variables $\Delta, \delta, \tau$, and $\varepsilon$ are defined in Fig. 1. When $\zeta=0$, the signal is encoded for diffusion in the $z$ direction of the gradient frame of reference shown in Fig. 1 (bottom right). Diffusion in this single direction contains contributions from both the isotropic and anisotropic parts of the diffusion tensor, and we will denote such diffusion encoding as "directional." Isotropic encoding is achieved by setting $\zeta$ to the "magic-angle" $\operatorname{acos}\left(1 / 3^{1 / 2}\right) \approx 54.74^{\circ}$ [59]. This case is analogous to the solid-state NMR technique magic-angle (a)

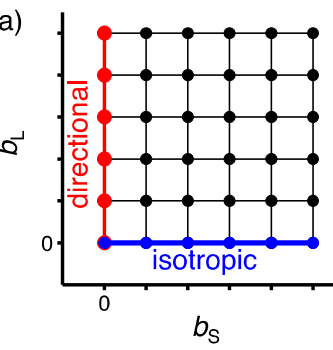

(b)

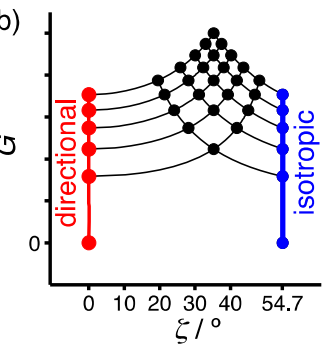

FIG. 2. Data sampling strategy for $2 \mathrm{D}$ correlation of isotropic and directional diffusion using the pulse sequence in Fig. 1. (a) 2D space spanned by the linear $b_{\mathrm{L}}$ and spherical $b_{\mathrm{S}}$ components of the diffusion-encoding tensor $\mathbf{b}$ as defined in Eq. (3). Isotropic (blue) and directional (red) encoding correspond to the colored perpendicular lines in the $\left(b_{\mathrm{L}}, b_{\mathrm{S}}\right)$ space. (b) 2D mesh of gradient amplitudes $G$ and angles $\zeta$ calculated from the square grid in the $\left(b_{\mathrm{L}}, b_{\mathrm{S}}\right)$ space by inverting Eqs. (9) and (10).

hopping [60], wherein information about the isotropic chemical shifts is obtained by rotating the sample in three discrete steps.

Using the relations between the $b$-tensor elements and the angle $\zeta$ given in Ref. [59], it can be shown that within the range $0 \leq \zeta<54.74^{\circ}$, the $b$-tensor components $b_{\mathrm{L}}, b_{\mathrm{P}}$, and $b_{\mathrm{S}}$ can be written as

$$
\begin{aligned}
& b_{\mathrm{L}}=b P_{2}(\cos \zeta), \\
& b_{\mathrm{P}}=0, \\
& b_{\mathrm{S}}=b\left[1-P_{2}(\cos \zeta)\right],
\end{aligned}
$$

where $P_{2}(x)=\left(3 x^{2}-1\right) / 2$ is the second Legendre polynomial. According to Eqs. (9) and (10), the $2 \mathrm{D}\left(b_{\mathrm{L}}, b_{\mathrm{S}}\right)$ space can be sampled through the variables $G$ and $\zeta$ that are under direct experimental control. Figure 2(a) shows the conceptually simplest way of sampling the $\left(b_{\mathrm{L}}, b_{\mathrm{S}}\right)$ space: a square grid with an equal number of points in both dimensions. By inverting Eqs. (9) and (10), we can calculate the corresponding 2D mesh in $(G, \zeta)$ space as shown in Fig. 2(b).

The new diffusion NMR method is demonstrated using a composite sample with three water components resembling the ones that have been used to model water diffusion in chemically fixed rat brain [61]. As illustrated in Fig. 3, the sample is a $5 \mathrm{~mm}$ NMR tube, with a lyotropic liquid crystal, placed inside a $10 \mathrm{~mm}$ tube filled with a yeast cell suspension [37]. The liquid crystal was prepared as described in Ref. [38] using the composition 45 wt \% water (Milli-Q quality), $40 \mathrm{wt} \%$ of the hydrocarbon 2,2,4-trimethylpentane (Sigma-Aldrich, Sweden), and $15 \mathrm{wt} \%$ of the detergent sodium 1,4-bis(2-ethylhexoxy)1,4-dioxobutane-2-sulfonate (trade name AOT from Sigma-Aldrich, Sweden). According to the phase diagram in Ref. [62], the liquid crystal is of the reverse 2D hexagonal type, giving rise to pronounced diffusion anisotropy of the water [38]. The diffusion properties of 


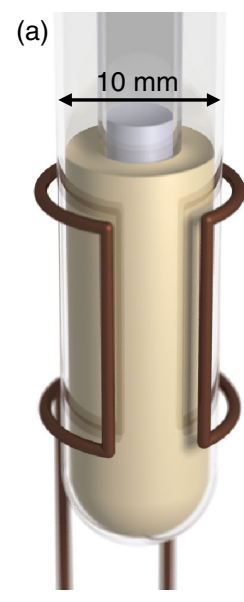

(b)

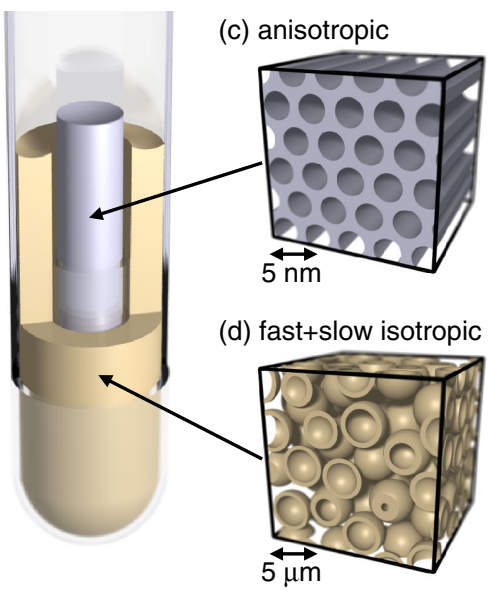

FIG. 3. Schematics of geometry and microstructure of a composite yeast cell suspension and liquid crystal sample giving three distinct water components. (a) Assembled sample with coaxial glass tubes located in a $10 \mathrm{~mm}$ rf coil. (b) Cutaway view revealing the geometries of the solutions in the inner and outer tubes with outer diameters of 5 and $10 \mathrm{~mm}$, respectively. (c) Magnification of a section of a single microdomain in the polydomain liquid crystal in the inner tube. (d) Micrometer-scale structure of the yeast cell suspension in the $10 \mathrm{~mm}$ tube. The spherical shells symbolize the cell membranes that separate the intra- and extracellular water.

water in the liquid crystal resemble intra-axonal water in tissue regions with low, but nonzero, orientational order. The yeast cell suspension was prepared as reported in Ref. [37]. Two water populations can be distinguished in the cell suspension, an intracellular and an extracellular one, both of them featuring isotropic diffusion but with a few orders of magnitude difference in apparent diffusivity $[36,44,63]$. The extracellular component resembles extraaxonal water or cerebrospinal fluid, while the intracellular component corresponds to the slow isotropic water that has been inferred from advanced model fitting of conventional diffusion MRI data [61].

Experiments were performed at $291.2 \mathrm{~K}$ on a Bruker Avance-II $500 \mathrm{MHz}$ spectrometer using a MIC-5 probe fitted with a $10 \mathrm{~mm}$ rf insert. Data were acquired with the timing variables $\delta=3.7 \mathrm{~ms}, \Delta=59.2 \mathrm{~ms}, \varepsilon=0.1 \mathrm{~ms}$, $\tau=0.2 \mathrm{~ms}, \tau_{1}=5.2 \mathrm{~ms}, \tau_{2}=54.0 \mathrm{~ms}, \tau_{\mathrm{e}}=22.0 \mathrm{~ms}$, and recycle delay $=2 \mathrm{~s}$, gradient amplitudes from 0.004 to $0.630 \mathrm{~T} / \mathrm{m}$ in 16 geometrically spaced increments, and angles $\zeta$ selected to give a rectangular sampling of the $\left(b_{\mathrm{L}}, b_{\mathrm{S}}\right)$ space. The data were powder averaged over 29 orientations of the gradient frame [37], thereby enforcing an isotropic orientation distribution as required for Eqs. (5) and (8) to be valid. A two-step rf pulse and receiver phase cycle and extensive use of spoiler gradients was used to assure that all acquired signal originates from the first $90^{\circ}$ pulse [38]. The total experiment time was $8.5 \mathrm{~h}$. The ${ }^{1} \mathrm{H}$ signal was recorded with $10 \mathrm{kHz}$ spectral width and 1024 complex time-domain points. After Fourier

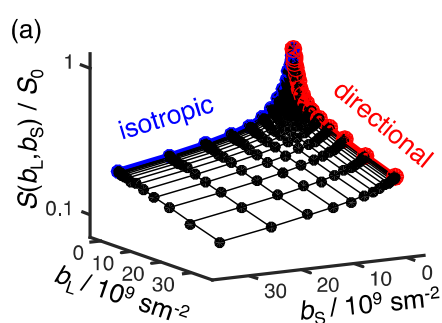

(b) $P\left(D_{z z}, D_{\text {iso }}\right)$
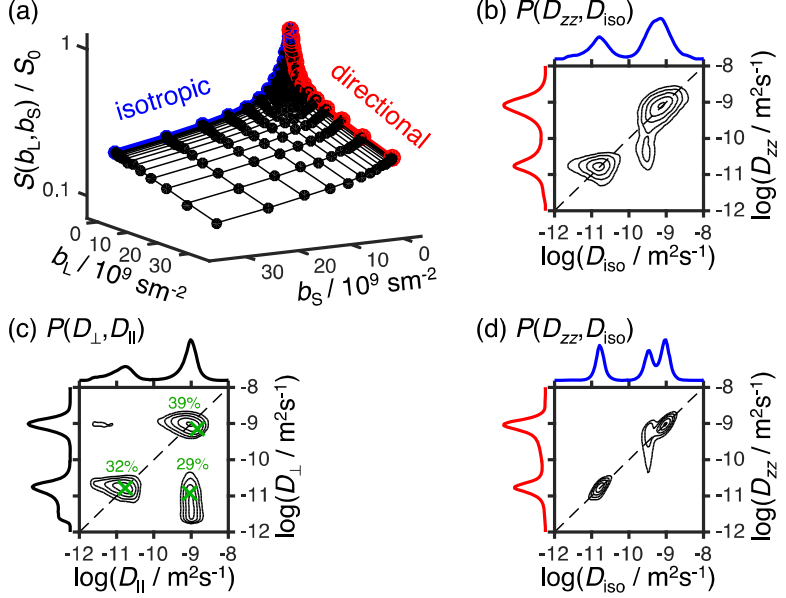

(d) $P$

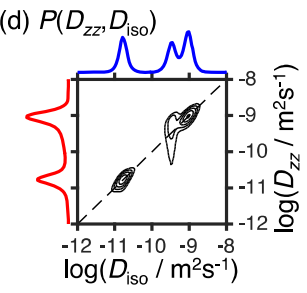

FIG. 4. 2D correlation of isotropic and directional water diffusion for the three-component sample in Fig. 3. (a) Water signal amplitude $S\left(b_{\mathrm{L}}, b_{\mathrm{S}}\right) / S_{0}$ sampled on a geometrically spaced rectangular grid in the $2 \mathrm{D}\left(b_{\mathrm{L}}, b_{\mathrm{S}}\right)$ space (circles, experimental; lines, calculated from the sparse basis analysis). The traces corresponding to purely isotropic and directional encoding are highlighted with the colors blue and red, respectively. (b) 2D directional and isotropic diffusion correlation spectrum $P\left(D_{z z}, D_{\text {iso }}\right)$ obtained by $2 \mathrm{D}$ inverse Laplace transformation. (c) $2 \mathrm{D}$ correlation spectrum of radial and axial diffusivities $P\left(D_{\perp}, D_{\|}\right)$estimated by numerical inversion of Eq. (7). The result of a three-component fit of Eq. (7) is indicated with green crosses and percentages for the relative weights of the components. (d) 2D spectrum $P\left(D_{z z}, D_{\text {iso }}\right)$ obtained from $P\left(D_{\perp}, D_{\|}\right)$in panel (c) by transformation according to Eq. (6). In (b)-(d), the contour lines are linearly spaced from $5 \%$ to $90 \%$ of the maximum value and the dashed lines indicate the diagonals. The traces above and to the left of each contour plot represent the $1 \mathrm{D}$ projections of the $2 \mathrm{D}$ distributions onto the respective axis. Note that the three components are widely separated in the sparse basis in panel (c).

transformation, phase correction, and baseline correction, the water resonance line was integrated and stored for further analysis. All data processing was performed with in-house code written in MATLAB (The Mathworks, Natick, MA).

The experimental results are shown in Fig. 4. The traces labeled "isotropic" and "directional" in Fig. 4(a) highlight the fact that these two sets of 1D data form orthogonal lines in the $2 \mathrm{D}\left(b_{\mathrm{L}}, b_{\mathrm{S}}\right)$ space. The distribution $P\left(D_{z z}, D_{\text {iso }}\right)$ was estimated by subjecting the acquired data to a numerical 2D ILT using the method of English et al. [55] augmented with a bootstrapping procedure $[64,65]$ to generate $10^{4}$ realizations of $P\left(D_{z z}, D_{\text {iso }}\right)$. Averaging of all these realizations was used to reduce the influence of spurious features [65], giving a smooth $2 \mathrm{D}$ spectrum $P\left(D_{z z}, D_{\text {iso }}\right)$ as shown in Fig. 4(b). In this representation, an isotropic component is located on the diagonal $\left(D_{z z}=D_{\text {iso }}\right)$, while an anisotropic component gives rise to a ridge in parallel with the $D_{z z}$ axis since a unique value of $D_{\text {iso }}$ is correlated with a whole range of values of $D_{z z}$ that, according to Eq. (5), 
depend on the eigenvalues of the diffusion tensor as well as its orientation with respect to the $b$ tensor. While a slow isotropic component, corresponding to the intracellular water of the yeast suspension, is easily discerned at $D_{\text {iso }}=D_{z z} \approx 10^{-11} \mathrm{~m}^{2} \mathrm{~s}^{-1}$, there is no clear separation between the two remaining ones with $D_{\text {iso }}$ on the order of $10^{-9} \mathrm{~m}^{2} \mathrm{~s}^{-1}$.

Improved resolution of the three components can be obtained by numerical inversion of Eq. (7) using the same procedure as for the $2 \mathrm{D}$ ILT above. The resulting distribution $P\left(D_{\perp}, D_{\|}\right)$in Fig. 4(c) features three clearly separated components: two isotropic ones at $D_{\|}=$ $D_{\perp} \approx 10^{-11}$ and $10^{-9} \mathrm{~m}^{2} \mathrm{~s}^{-1}$, corresponding to the intraand extracellular water, as well as an anisotropic component with $D_{\|} \approx 10^{-9} \mathrm{~m}^{2} \mathrm{~s}^{-1}$ and $D_{\perp} \approx 10^{-11} \mathrm{~m}^{2} \mathrm{~s}^{-1}$ originating from the liquid crystal. The green crosses in Fig. 4(c) show the values of $D_{\|}$and $D_{\perp}$ determined by fitting a three-component version of Eq. (7) to the data. As described in the Supplemental Material [45], the measured apparent diffusivities $D_{\|}=D_{\perp}=1.7 \times 10^{-11} \mathrm{~m}^{2} \mathrm{~s}^{-1}$ for the intracellular component are consistent with restricted diffusion in a spherical cell with a radius between 2.7 and $3.0 \mu \mathrm{m}$, an intracellular water diffusivity of $0.56 \times$ $10^{-9} \mathrm{~m}^{2} \mathrm{~s}^{-1}$ [44], and the herein used pulse sequence parameters. The cell radius is in reasonable agreement with the literature data for yeast cells $[44,66,67]$.

In order to keep the analogy with the corresponding solid-state NMR experiments, numerical evaluation of Eq. (6) can be used to convert $P\left(D_{\perp}, D_{\|}\right)$to the $\left(D_{z z}, D_{\text {iso }}\right)$ basis as shown in Fig. 4(d). Comparison between Figs. 4(b) and 4(d) verifies that the indirect route via the sparse representation in the $\left(D_{\perp}, D_{\|}\right)$basis gives a more clear separation of the liquid crystal and extracellular components. In the latter case, the intra- and extracellular components both lie on the diagonal, and the liquid crystal component is a ridge perfectly aligned with the $D_{z z}$ axis, spanning both sides of the diagonal.

We have suggested and demonstrated a diffusion NMR experiment that can resolve subensembles of water with distinct coordinates in a sparse 2D basis of radial and axial diffusivities. Our recent diffusion MRI methods for in vivo mapping of microscopic diffusion anisotropy [68] already sample two perpendicular $1 \mathrm{D}$ lines in the $2 \mathrm{D}$ acquisition space of the new method, and can readily be adapted to sample the full 2D space [54]. In line with the path of development of our method for quantifying water diffusional exchange in the human brain [12,57,69-71], we plan to carefully select a sparse set of informative data points in the herein densely sampled 2D acquisition space, thereby permitting measurements within the time frame of clinical MRI. By imposing physiologically reasonable constraints on the values of the components, the sparsely sampled data can then be converted to populations and local diffusivities that are not only valuable by themselves, but could also serve as input parameters for the current algorithms to recover the orientation distributions of the anisotropic components [34].

This work is financially supported by the Swedish Foundation for Strategic Research (No. AM13-0090) and the Swedish Research Council (No. 2009-6794, No. 2014-3910). Dan Lundberg (CR Competence AB, Lund, Sweden) is gratefully acknowledged for suggesting the liquid crystal used in this work.

*Corresponding author. joao.martins@fkem1.lu.se

[1] W.S. Price, NMR Studies of Translational Motion (Cambridge University Press, Cambridge, 2009).

[2] P. T. Callaghan, Translational Dynamics and Magnetic Resonance (Oxford University Press, Oxford, 2011).

[3] D. Le Bihan, Nat. Rev. Neurosci. 4, 469 (2003).

[4] Y. Assaf, T. Blumefeld-Katzir, Y. Yovel, and P. J. Basser, Magn. Reson. Med. 59, 1347 (2008).

[5] P. J. Basser, J. Mattiello, and D. Le Bihan, Biophys. J. 66, 259 (1994).

[6] D. S. Tuch, T. G. Reese, M. R. Wiegell, N. Makris, J. W. Belliveau, and V. J. Wedeen, Magn. Reson. Med. 48, 577 (2002).

[7] E. O. Stejskal and J. E. Tanner, J. Chem. Phys. 42, 288 (1965)

[8] N. Shemesh et al., Magn. Reson. Med. 75, 82 (2016).

[9] D. G. Cory, A. N. Garroway, and J. B. Miller, Polym. Prepr. 31, 149 (1990).

[10] P. P. Mitra, Phys. Rev. B 51, 15074 (1995).

[11] Y. Cheng and D. G. Cory, J. Am. Chem. Soc. 121, 7935 (1999).

[12] P. T. Callaghan and I. Furó, J. Chem. Phys. 120, 4032 (2004).

[13] Y. Qiao, P. Galvosas, and P. T. Callaghan, Biophys. J. 89, 2899 (2005).

[14] M. A. Koch and J. Finsterbusch, Magn. Reson. Med. 60, 90 (2008).

[15] J. Finsterbusch and M. A. Koch, J. Magn. Reson. 195, 23 (2008)

[16] E. Özarslan and P. J. Basser, J. Chem. Phys. 128, 154511 (2008).

[17] T. Weber, C. H. Ziener, T. Kampf, V. Herold, W. R. Bauer, and P. M. Jakob, Magn. Reson. Med. 61, 1001 (2009).

[18] E. Özarslan, J. Magn. Reson. 199, 56 (2009).

[19] J. Finsterbusch, Annual Reports on NMR Spectroscopy 72, 225 (2011).

[20] N. Shemesh, T. Adiri, and Y. Cohen, J. Am. Chem. Soc. 133, 6028 (2011).

[21] N. Shemesh and Y. Cohen, Magn. Reson. Med. 65, 1216 (2011)

[22] S. N. Jespersen, H. Lundell, C. K. Sønderby, and T. B. Dyrby, NMR Biomed. 26, 1647 (2013).

[23] N. Shemesh, E. Özarslan, M. E. Komlosh, P. J. Basser, and Y. Cohen, NMR Biomed. 23, 757 (2010).

[24] M. Lawrenz and J. Finsterbusch, Magn. Reson. Med. 69, 1072 (2013). 
[25] N. Shemesh, J. T. Rosenberg, J. N. Dumez, J. A. Muniz, S. C. Grant, and L. Frydman, Nat. Commun. 5, 4958 (2014).

[26] G. J. Stanisz, A. Szafer, G. A. Wright, and R. M. Henkelman, Magn. Reson. Med. 37, 103 (1997).

[27] T. E. Behrens, M. W. Woolrich, M. Jenkinson, H. JohansenBerg, R. G. Nunes, S. Clare, P. M. Matthews, J. M. Brady, and S. M. Smith, Magn. Reson. Med. 50, 1077 (2003).

[28] Y. Assaf, R. Z. Freidlin, G. K. Rohde, and P. J. Basser, Magn. Reson. Med. 52, 965 (2004).

[29] M. Nilsson, D. van Westen, F. Ståhlberg, P. C. Sundgren, and J. Lätt, Magnetic Resonance Materials in Physics, Biology, and Medicine 26, 345 (2013).

[30] O. Pasternak, N. Sochen, Y. Gur, N. Intrator, and Y. Assaf, Magn. Reson. Med. 62, 717 (2009).

[31] U. Ferizi, T. Schneider, E. Panagiotaki, G. Nedjati-Gilani, H. Zhang, C. A. Wheeler-Kingshott, and D. C. Alexander, Magn. Reson. Med. 72, 1785 (2014).

[32] J. D. Tournier, F. Calamante, D. G. Gadian, and A. Connelly, NeuroImage 23, 1176 (2004).

[33] F. Dell'Acqua, G. Rizzo, P. Scifo, R. A. Clarke, G. Scotti, and F. Fazio, IEEE Trans. Biomed. Eng. 54, 462 (2007).

[34] B. Jeurissen, A. Leemans, J. D. Tournier, D. K. Jones, and J. Sijbers, Hum. Brain Mapp. 34, 2747 (2013).

[35] K. Schmidt-Rohr and H. W. Spiess, Multidimensional Solid-State NMR and Polymers (Academic Press, San Diego, 1994).

[36] S. Eriksson, S. Lasič, and D. Topgaard, J. Magn. Reson. 226, 13 (2013).

[37] S. Lasič, F. Szczepankiewicz, S. Eriksson, M. Nilsson, and D. Topgaard, Front. Phys. 2, 11 (2014).

[38] S. Eriksson, S. Lasič, M. Nilsson, C.-F. Westin, and D. Topgaard, J. Chem. Phys. 142, 104201 (2015).

[39] D. E. Woessner, J. Phys. Chem. 67, 1365 (1963).

[40] J. E. Tanner, J. Chem. Phys. 69, 1748 (1978).

[41] P. P. Mitra, P. N. Sen, L. M. Schwartz, and P. Le Doussal, Phys. Rev. Lett. 68, 3555 (1992).

[42] L. L. Latour, P. P. Mitra, R. L. Kleinberg, and C. H. Sotak, J. Magn. Reson., Ser. A 101, 342 (1993).

[43] F. Stallmach, J. Kärger, C. Krause, M. Jeschke, and U. Oberhagemann, J. Am. Chem. Soc. 122, 9237 (2000).

[44] I. Åslund and D. Topgaard, J. Magn. Reson. 201, 250 (2009).

[45] See Supplemental Material http://link.aps.org/supplemental/ 10.1103/PhysRevLett.116.087601, which includes Refs. [46-49], for a detailed calculation of the intracellular radius, and a study on the range of validity of the multiGaussian approach.

[46] C. H. Neuman, J. Chem. Phys. 60, 4508 (1974).

[47] D. C. Douglass and D. W. McCall, J. Phys. Chem. 62, 1102 (1958).

[48] J. S. Murday and R. M. Cotts, J. Chem. Phys. 48, 4938 (1968).

[49] P. Linse and O. Söderman, J. Magn. Reson., Ser. A 116, 77 (1995).
[50] A. Bax, N. M. Szeverenyl, and G. E. Maciel, J. Magn. Reson. 55, 494 (1983).

[51] R. C. Ziegler, R. A. Wind, and G. E. Maciel, J. Magn. Reson. 79, 299 (1988).

[52] L. Frydman, G. C. Chingas, Y. K. Lee, P. J. Grandinetti, M. A. Eastman, G. A. Barrall, and A. Pines, J. Chem. Phys. 97, 4800 (1992).

[53] C.-F. Westin, F. Szczepankiewicz, O. Pasternak, E. Özarslan, D. Topgaard, H. Knutsson, and M. Nilsson, Med. Image Comput. Comput. Assist. Interv. 8675, 209 (2014).

[54] J. Sjölund, F. Szczepankiewicz, M. Nilsson, D. Topgaard, C.-F. Westin, and H. Knutsson, J. Magn. Reson. 261, 157 (2015).

[55] A. E. English, K. P. Whittal, M. L. G. Joy, and R. M. Henkelman, Magn. Reson. Med. 22, 425 (1991).

[56] Y.-Q. Song, L. Venkataramanan, M. D. Hürlimann, M. Flaum, P. Frulla, and C. Straley, J. Magn. Reson. 154, 261 (2002).

[57] D. Bernin and D. Topgaard, Curr. Opin. Colloid Interface Sci. 18, 166 (2013).

[58] J. Mitchell, T. C. Chandrasekera, and L. F. Gladden, Prog. Nucl. Magn. Reson. Spectrosc. 62, 34 (2012).

[59] D. Topgaard, Microporous Mesoporous Mater. 205, 48 (2015).

[60] N. M. Szeverenyi, A. Bax, and G. E. Maciel, J. Magn. Reson. 61, 440 (1985).

[61] E. Panagiotaki, T. Schneider, B. Siow, M. G. Hall, M. F. Lythgoe, and D.C. Alexander, NeuroImage 59, 2241 (2012).

[62] B. Jönsson, B. Lindman, K. Holmberg, and B. Kronberg, Surfactants and Polymers in Aqueous Solution (John Wiley \& Sons Ltd, Chichester, 1998).

[63] M. D. Silva, K. G. Helmer, J.-H. Lee, S. S. Han, C. S. Springer Jr., and C. H. Sotak, J. Magn. Reson. 156, 52 (2002).

[64] B. Efron, Biometrika 68, 589 (1981).

[65] D. W. de Kort, J. P. van Duynhoven, F. J. Hoeben, H. M. Janssen, and H. Van As, Anal. Chem. 86, 9229 (2014).

[66] N. Shemesh, E. Özarslan, P. J. Basser, and Y. Cohen, NMR Biomed. 25, 236 (2012).

[67] N. Shemesh, G. A. Alvarez, and L. Frydman, PLoS One 10, e0133201 (2015).

[68] F. Szczepankiewicz, S. Lasič, D. van Westen, P. C. Sundgren, E. Englund, C.-F. Westin, F. Ståhlberg, J. Lätt, D. Topgaard, and M. Nilsson, NeuroImage 104, 241 (2015).

[69] I. Åslund, A. Nowacka, M. Nilsson, and D. Topgaard, J. Magn. Reson. 200, 291 (2009).

[70] S. Lasič, M. Nilsson, J. Lätt, F. Ståhlberg, and D. Topgaard, Magn. Reson. Med. 66, 356 (2011).

[71] M. Nilsson, J. Lätt, D. van Westen, S. Brockstedt, S. Lasič, F. Ståhlberg, and D. Topgaard, Magn. Reson. Med. 69, 1573 (2013). 